\title{
La ville, une expérience d'enseignement transversal au lycée (2016-2017)
}

The city, a cross-disciplinary teaching experience in High school (2016-2017)

\section{Fanny Letissier}

\section{(2) OpenEdition}

\section{Journals}

Édition électronique

URL : http://journals.openedition.org/trema/6277

DOI : $10.4000 /$ trema.6277

ISSN : 2107-0997

Éditeur

Faculté d'Éducation de l'université de Montpellier

Référence électronique

Fanny Letissier, «La ville, une expérience d'enseignement transversal au lycée (2016-2017) 》, Tréma [En ligne], 54 | 2020, mis en ligne le 01 décembre 2020, consulté le 14 décembre 2020. URL : http:// journals.openedition.org/trema/6277; DOI : https://doi.org/10.4000/trema.6277

Ce document a été généré automatiquement le 14 décembre 2020.

Trema 


\title{
La ville, une expérience d'enseignement transversal au lycée (2016-2017)
}

The city, a cross-disciplinary teaching experience in High school (2016-2017)

\author{
Fanny Letissier
}

\section{Introduction}

1 L'enseignement de l'histoire, de la géographie et de l'éducation morale et civique (EMC) dans le secondaire est réalisé par un même enseignant. La transversalité pourrait alors être efficacement envisagée. Cependant, le processus d'enseignement peine à produire des savoirs disciplinaires décloisonnés. A la lecture des programmes de l'Éducation nationale ${ }^{1}$, il est demandé aux professeurs de respecter "la parité horaire entre l'histoire et la géographie ». Dès lors, est-il intéressant de s'interroger sur l'intérêt d'envisager un enseignement séparé ?

2 En outre, la complexité (Morin, 2014) semble hors-champ de ces mêmes programmes. Il appert toutefois que les apports de la théorie de Morin permettent de penser la complexité du monde contemporain en l'intégrant dans les contenus scolaires. En ce sens, la complexité est envisageable et reste un objectif à atteindre en classe. Il s'agit alors d'analyser le réel en partant de l'empirie et en l'appréciant de manière transdisciplinaire. Comment l'envisager concrètement avec des lycéens?

Chercheur en géographie depuis une vingtaine d'années, j'étudie des phénomènes urbains. La complexité inhérente à l'objet - ville - parait difficilement appréhendable par des élèves de seconde. Pourtant, le point de départ de cette démarche a consisté à utiliser des outils et des méthodes employés d'ordinaire par des chercheurs chevronnés. Le thème de l'urbain pourrait être l'objet de maintes analyses simplificatrices, mais l'expérience menée en classe de seconde en 2016-2017 a prouvé 
que la complexité pouvait être envisagée en choisissant une approche décloisonnée mettant en lien les trois enseignements dispensés.

Après nous être interrogés sur la manière d'appréhender l'objet - ville - en classe de seconde, nous développerons le protocole didactique employé sur l'année. Enfin, nous présenterons la méthode et les résultats obtenus et nous élargirons à d'autres projets possibles liant complexité et transversalité.

\section{La démarche. Appréhender la ville en tant qu'objet complexe}

5 S'interroger sur la manière d'appréhender la ville comme objet complexe revient à identifier une démarche qui allie efficacement l'histoire, la géographie et l'EMC. Enseignants et élèves sont habitués à vivre le cloisonnement disciplinaire dans leur vie scolaire. Quel cheminement permet d'aborder la complexité de la ville avec les élèves?

\section{Une démarche décloisonnée}

6 Enseigner la ville n'est pas une pratique qui m'est étrangère ${ }^{2}$, mais la transposition didactique attendue dans le cadre actuel présente plusieurs difficultés de prime abord. Une réflexion autour de la question de la ville s'est développée afin de l'aborder sur l'année entière, de manière décloisonnée. L'étude de la ville ne s'arrête pas à un moment d'histoire, même si chaque ville étudiée correspond à un moment particulier, reflétant par conséquent, une urbanité singulière. Histoire et géographie sont intimement liées dans l'étude des villes. "L'histoire emprunte à la géographie (les lieux, l'espace) et la géographie à l'histoire (faite d'histoire et d'histoires) » (Audigier, 1997, p.54). Afin de pouvoir parler d'espace tendant vers une durabilité, il est nécessaire d'analyser la fabrication urbaine, tant en termes de substance et d'interactions sociales qu'en terme matériel, sur un temps long (Letissier, 2007). Sans chercher à simplifier la complexité (Morin, 1990), comment passer d'un discours géographique produit dans un cadre scientifique à un discours didactique visant une propédeutique (Thémines, 2006)? Réaliser des ponts entre l'histoire, la géographie et l'EMC apparaît comme une démarche évidente à mettre en place avec les élèves. Par conséquent, chaque séance abordant la ville dans l'une de ces disciplines, apporte un éclairage pertinent à l'espace urbain. Dès lors, l'élève appréhende mieux la ville dans sa globalité et sa complexité tout en établissant des liens disciplinaires.

\section{Déconstruire les lieux communs sur les disciplines historiques et géographiques}

7 Pour bon nombre de lycéens, chacun pense savoir ce qu'est la géographie ou l'histoire. Pourtant, au travers de discussions, on réalise rapidement la méprise sur le sujet. Il est utile de déconstruire les représentations erronées qui traversent la société contemporaine. La géographie n'est ni une nomenclature, ni une discipline où le savoir encyclopédique prime (Knafou, 1997). Par ailleurs, de nombreux élèves affirment ne pas apprécier certaines périodes historiques - le Moyen-âge ou l'Antiquité - estimant qu'ils en savent suffisamment sur ces périodes, déjà abordées au cours de leur scolarité. Présenter alors quelques résultats des recherches actuelles (Noizet \& Cadeddu, 2016) est utile pour balayer les lieux communs disciplinaires. Dans ce contexte, l'enjeu relève 
alors de la mise en place de méthodes qui permettent à l'élève de découvrir des raisonnements géographiques et historiques complexes, sur le principe de géographicité (Thémines, 2006) et d'historicité (Hartog, 2003) - étant entendu qu'il s'agit ici du rapport que l'élève entretient avec l'espace en général d'une part, les temporalités longues d'autre part.

\section{Vers un socle commun}

8 L'expérience proposée s'inscrit dans une démarche d'enseignement des sciences sociales. Il s'agit de mettre en place une discipline du social grâce aux dimensions historique et spatiale avec des concepts bien spécifiques. Lévy proposait déjà en 1986 que l'on dispense dans les écoles, collèges et lycées un «enseignement intégré des sciences sociales » comprenant « une didactique du social et notamment de l'espace». Dès lors, la géographie, l'histoire et l'EMC pourraient se fondre légitimement au sein des sciences humaines et sociales. L'étude de la ville trouverait alors facilement sa place. Le rôle d'enseignant apparaît en ce sens comme facilitateur de la construction de l'édifice en proposant aux élèves le ciment qui lie les blocs entre eux (Frémont, 1986). Dès lors, il est préférable de conserver un horizon de compréhension des enjeux contemporains sociétaux, grâce à des outils d'intelligibilité du réel.

\section{Enseigner la complexité de la ville : le choix de l'approche conceptuelle}

9 La ville n'est pas un objet d'étude ordinaire. Son apparente banalité recèle une complexité intéressante à analyser avec des lycéens. En tant que "géotype de substance sociale basée sur la coprésence» (Lévy \& Lussault, 2004, p. 873), elle représente l'espace que la majorité des citoyens du monde habitent aujourd'hui.

10 Pendant très longtemps, l'enseignement de la ville s'est cantonné à indiquer des positions géographiques sur des cartes, à apprendre des fonctions urbaines, à retenir des paysages typiques (Allemand, Dagorn \& Vilaça, 2005). Il s'agissait alors d'une géographie nomenclaturale et d'une histoire datée, nourrie grâce au traité de Chabot sur les villes daté de 1948 (Thémines, 2006).

11 Le psychologue américain Bruner, cité par Audigier, affirmait dans les années 1970 que l'on pouvait appréhender une science de manière pertinente avec des élèves à condition de s'appuyer « sur son noyau dur, ses concepts essentiels et la nécessité d'une construction spiralaire des savoirs. (...)» (Audigier, 1997, p. 54). Dans le cadre urbain, cette démarche répond exactement au souci de se confronter à la complexité. C'est cette ligne de pensée qu'il a été choisi de suivre, en encadrant le concept de ville de manière à proposer différentes notions essentielles qui en appellent d'autres au fil des chapitres liés à la ville.

Lors d'une conférence ayant pour thème la définition de la ville, à destination d'enseignants du second degré, Dumont expose la difficulté d'enseigner la ville du fait de la diversité des situations statistiques nationales (Dumont, 2016). Pourtant, ces critères d'ordre statistique ne paraissent pas introduire une démarche où la complexité est sollicitée. En effet, on privilégie grâce au principe hologrammique, cher à Morin, un enseignement de la ville où l'étude des parties constituantes de cette-dernière est essentielle pour connaître la ville dans son ensemble. Penser la complexité est une 
chose nécessaire dans notre société (Morin, 2005). Roumégous confirme l'utilité de la transmission de la connaissance dans un cadre conceptuel problématisé et structuré. Faire le choix de pénétrer la ville par sa substance - étant entendu qu'elle est coconstruite par une multitude d'acteurs - plutôt que par des statistiques semble alors primordial : on aborde ainsi la ville par son urbanité - en associant la densité à la diversité (Lévy \& Lussault, 2004). Cette approche sous-entend que l'urbanité d'une ville est lisible à la fois dans le tout qu'elle forme et dans ses parties, comme les espaces publics qui la constituent.

\section{Une démarche fondée sur le « modèle vécu »}

Cette démarche s'inscrit par ailleurs dans la logique d'un enseignement au service du citoyen. En effet, il appert qu'étudier la ville tout au long de l'année à partir de «modèles vécus » (Vezier, 2013) grâce à l'EMC, introduit une complexité conceptuelle et situationnelle. En interrogeant en premier lieu les élèves sur leur manière de vivre la ville, ils prennent davantage conscience de leur statut de citadins-citoyens. Dans la mesure où les habitants progressent vers l'état de citadins grâce à leur stratégie spatiale, habiter une ville correspond à "s'identifier [et à] se rendre responsable" (Médam, 1998, p. 50). Ainsi, par le truchement du modèle vécu, les élèves expérimentent des concepts introduits en histoire et en géographie, limitant de fait l'approche disciplinaire. Enfin, l'EMC permet de confronter les élèves à des problèmes concrets, tels que des conflits d'usagers sur un espace partagé, et en leur offrant la possibilité d'être acteurs, porteurs de solutions: «c'est en citoyennant qu'on devient citoyen » (Audigier, 2012, p. 11).

\section{Le Protocole didactique. Construire le concept de ville}

Le protocole didactique est fondé sur la recherche d'une cohérence globale de la construction du concept de "ville» sur l'année. Le protocole construit répond au questionnement suivant : Comment le récit prospectif (en géographie/EMC) peut-il être mis en parallèle avec le récit historique? En ce sens, comment passer du passé au futur? Le tableau suivant présente une proposition de mise en œuvre de l'approche du concept de ville sur l'année.

Tableau synthétique de la démarche adoptée sur l'année ${ }^{3}$

\begin{tabular}{|l|l|l|l|l|l|}
\hline & Étape 1 & Étape 2 & Étape 3 & Étape 4 & Étape 5 \\
\hline $\begin{array}{l}-\quad \text { Nombre } \\
\text { de } \\
\text { séquences }\end{array}$ & 1 séquence & 1 séquence & 2 séquences & 2 séquences & $\begin{array}{l}1 \\
\text { séquence }\end{array}$ \\
\hline
\end{tabular}




\begin{tabular}{|c|c|c|c|c|c|}
\hline $\begin{array}{l}\text { - Thème } \\
\text { abordé }\end{array}$ & $\begin{array}{lr}\text { Étude } & \text { d'un } \\
\text { espace } & \text { public } \\
\text { ordinaire } & \text { à } \\
\text { partir } & \text { d'une } \\
\text { photographie. }\end{array}$ & $\begin{array}{ll}\text { Étude de la } \\
\text { ville pendant } \\
\text { l'Antiquité à } \\
\text { travers } & \\
\text { l'analyse de } \\
\text { l'agora } & \\
\text { orecaue } & \end{array}$ & $\begin{array}{l}\text { Étude de deux villes } \\
\text { reflétant } \\
\text { urbanité contrastée } \\
\text { (Londres une } \\
\text { Johannesburg). }\end{array}$ & $\begin{array}{l}\text { Exercice de } \\
\text { Prospective: } \\
\text { L'espace pratiqué } \\
\text { et souhaité des } \\
\text { usagers situé } \\
\text { devant le lycée. }\end{array}$ & $\begin{array}{l}\text { Étude de } \\
\text { la ville au } \\
\text { Moyen- } \\
\text { Age. }\end{array}$ \\
\hline
\end{tabular}

\section{Des élèves acteurs de leur ville en EMC}

L'entrée en matière se réalise par l'analyse des règles de l'espace public urbain en EMC. Partir d'espaces sociaux concrets est une démarche à privilégier avec les élèves. L'espace public est un excellent vecteur de compréhension globale de la ville : la société urbaine y est visible et les élèves ont plus facilement accès à la ville et à la société qui la construit. Un jeu de rôle est prévu au cours des séances d'EMC. Les élèves endossent le rôle de citoyens dans un processus fictif de concertation avec les élus pour réaménager un espace public urbain. Dans ce dessein, il appert que l'on peut partir de l'opposition "ville conçue, ville vécue » que mettait en avant Lefebvre afin de lever le voile sur le possible décalage entre les conceptions urbaines des urbanistes et les pratiques effectivement réalisées par les citadins (Lefebvre, 2000). Cette étude permet ainsi d'anticiper sur le chapitre de géographie "aménager la ville» dans le cadre de l'exploration de la dimension prospective de la géographie, requise dans les programmes.

Chacun est plus qu'un actant dans sa vie de citadin (Lussault, 2007), c'est bien en cela que les élèves sont de futurs citoyens, acteurs de leurs pratiques et de leurs souhaits d'aménagement d'espace pratiqué au quotidien. Ils appartiennent à la société qui fonctionne grâce aux interactions entre ses différents acteurs (Elias, 1991). Par conséquent, il est important que les deux niveaux - individuel et sociétal - soient mentionnés lors de la mise en place des séquences d'EMC.

\section{Mettre en évidence la continuité historique en matière de citoyenneté urbaine}

17 Le programme d'histoire permet d'aborder la ville à deux périodes différentes de l'année. Dans un premier temps, elle est approchée de manière périphérique ${ }^{4}$. L'étude de l'agora grecque constitue en effet une base pour l'exercice de réflexion sur l'espace public en EMC. La mise en parallèle entre le récit prospectif et le récit historique débute donc à cette période. Ainsi, pour passer du passé au futur, L'EMC permet tout au long de l'année de conserver le questionnement tout en l'ancrant au sein d'une réalité concrète et présente. L'enjeu consiste avant tout à permettre aux adolescents de réaliser des liens entre ces parties de cours particulièrement éloignées historiquement.

Dans un second temps, l'objectif du chapitre d'histoire "Sociétés et cultures urbaines " au sein de ce protocole contribue à définir l'objet ville, en schématisant sa fabrication (Mumford, 1961). Les attributs du concept pourront être utilisés alors qu'ils ont déjà été définis plus tôt pour la ville contemporaine en géographie. 


\section{La ville durable en géographie}

Les sciences sociales emploient des modèles théoriques qui peuvent servir à analyser un espace urbain par exemple. "Comment définir la ville durable? " Cette question n'est pas simple (Lussault, 2011). Le choix est fait de comparer deux modèles urbains qui font partie intégrante des écrits de Lévy : le modèle de Johannesburg et le modèle d'Amsterdam (Lévy, 2010). À partir de ces cas exemplaires, les élèves s'interrogent sur l'existence de villes durables dans le monde et en analysent la pluralité.

\section{La méthode. Mettre en œuvre la complexité au lycée à partir d'une approche transversale}

Les élèves sont des individus à prendre dans leur globalité, en prenant en compte leur subjectivité et non comme des réceptacles de savoir disciplinaire (Audigier, 1997). C'est dans cette perspective qu'une approche transversale de la ville est mise en place sur l'année.

\section{La séquence d'EMC : pratiquer l'espace public}

21 L'approche de la ville débute par une étude d'espace public en EMC qui représente une échelle d'analyse idoine. En effet, son intérêt réside dans son aptitude à représenter la société urbaine observée. Il est à ce titre un condensé d'urbanité qui affiche une certaine manière d'associer la densité à la diversité (Letissier, 2007). En outre, c'est une entrée qui permet d'aborder la ville à partir d'un espace facilement identifiable par les élèves.

\section{Étape 1 : les règles de l'espace public}

Lors de la première séance, une photographie d'espace public est présentée aux élèves ${ }^{5}$. Ils répertorient ce que l'on peut trouver dans un espace public, que ce soit des objets ou des pratiques possibles. Cela permet de définir ce qu'il est envisageable de faire en tant que citoyen fréquentant un espace public. Ils prennent conscience que l'espace public urbain n'est pas un espace de liberté ordinaire (Paquot, 2009). Différents usages s'y côtoient. De ce fait, des règles de civilité s'y imposent.

\section{Étape 2 : l'exercice de prospective ${ }^{6}$}

Les élèves réalisent un dessin de toutes leurs pratiques de l'espace public devant le lycée. Pour ce faire, on peut afficher une vue aérienne de l'espace concerné, afin que chaque élève apprécie une vue d'ensemble du lieu et qu'ils n'en oublient aucun sousespace. Dans un second temps, les élèves dessinent leurs souhaits d'aménagement (dans un futur proche tout en respectant les piliers du Développement Durable additionnés aux dimensions politiques, religieuses, culturelles...) de cet espace afin que leurs pratiques soient améliorées. Chaque dessin devait être accompagné d'une légende et d'un court texte. Dans un troisième temps, les élèves participent à un jeu de rôle où ils endossent le rôle d'habitants-citoyens. Ils participent par conséquent à une réunion fictive de concertation pour le projet de réaménagement de l'espace public situé devant le lycée. Ils verbalisent alors leurs pratiques et leurs souhaits, dessinés au préalable. 
Une synthèse des souhaits les plus probants et redondants est réalisée afin de faire remonter des demandes précises à la mairie.

\section{La séquence de géographie : le choix de l'urbanité}

24 On débute la séquence de géographie en questionnant les élèves sur leurs représentations et leurs pratiques de l'espace urbain. En ce sens, partir d'un "modèle vécu » pour chercher à comprendre la ville des adolescents nous amène donc à réfléchir à la question de l'urbain en général et plus spécifiquement de l'urbanité. Les idées sont ensuite classées en trois grandes thématiques, relatives à : ce qu'il y a en ville, ce qu'est la ville, ce qu'on fait en ville $e^{7}$. De nombreux élèves vivent dans un territoire périurbain proche et ont réfléchi à leurs pratiques, avant de répondre s'ils se considéraient urbains ou non. Dès lors, la question de l'urbanité a été soulevée. Pour ce faire, les définitions de la densité et de la diversité ont été données aux élèves grâce aux éléments qu'ils ont énoncés au préalable. En outre, la manière dont la ville se construit morphologiquement, matériellement et de manière immatérielle est envisagée. En faisant entrer l'urbanité comme un patrimoine à conserver, les élèves associent les interactions sociales comme créant une forme particulière de ville au cours du temps.

Deux villes sont choisies, conformément au programme de seconde, Londres et Johannesburg, afin de représenter une ville des Nords et une ville des Suds ${ }^{8}$. On part d'une brève analyse de photos des villes choisies depuis une vue aérienne apposée à une vue en immersion dans un espace public. En effet, la ville peut être considérée, soit comme un lieu (un point d'un réseau ou un point sur un vaste territoire), soit comme une aire (un ensemble de lieux), utilisant ainsi une approche en extension et une approche en compréhension (Retaillé, 1997). On complète avec un plan de la ville et une carte présentant l'espace métropolitain inséré dans un réseau urbain d'un niveau scalaire supérieur. Les photos représentent deux villes différentes, symboles de deux types de sociétés. Le lien entre le paysage et la société fait ainsi écho au chapitre d'histoire médiévale. Un paysage n'est pas uniquement un espace observé, mais un espace qui se construit à travers des choix de société réels, à un moment donné. Nous avons ainsi réfléchi à la mesure de la densité et de la diversité, notamment à partir d'éléments aisément observables et concrets. Cela nous indiquait déjà une piste de compréhension du fonctionnement de chacune de ces deux villes.

Johannesburg est un espace hautement ségrégué, encore aujourd'hui. En ce sens, la célèbre remarque de Foucher - "l'apartheid se voit d'avion " - est toujours actuelle. Londres reflète l'urbanité européenne avec notamment une densité forte dans les quartiers centraux impliquant des choix sociétaux stratégiques en matière de mobilités. La ville a une histoire, elle se construit sur le long terme. Un détour par l'approche géohistorique (Braudel, 1969) aide à faire prendre conscience aux élèves que Johannesburg et Londres ne sont pas dans le même «moment géographique » et que l'héritage laissé à la ville aujourd'hui s'entretient, ou pas, pour atteindre une forme de durabilité sociétale. L'urbanité est donc au cœur de ce chapitre. Elle laisse émerger l'idée qu'il existe différents modèles urbains, notamment en termes d'héritage et de durabilité. Il s'agit donc de mesurer cette urbanité, à partir des critères ${ }^{9}$ proposés par Lévy. 


\section{La séquence d'histoire : concevoir une société urbaine} vue particuliers, est intéressant à envisager. En effet, une brève analyse des discours des élèves témoigne de la richesse de la démarche employée. Certains ont fait part d'un réel investissement pendant les séquences d'EMC et ont expliqué qu'il leur tenait à cœur de réaliser ce genre d'expérience où leur subjectivité est particulièrement exposée. En effet, lors des exercices d'EMC, les élèves peuvent révéler des dimensions de leur être, peu mises en avant habituellement. Leur sensibilité, leurs émotions, l'apprentissage spatial à travers leur corps, toutes ces expériences peuvent être exposées aux autres élèves et des échanges ont pu en découler. Ces apprentissages semblent avoir produit un substrat sur lequel les élèves ont pu engager des débats sur la manière d'organiser l'aménagement de la place située devant le lycée. La démarche engagée révèle en outre que les élèves ont acquis les bases d'un raisonnement géographique et historique tout en avançant sur la voie de la citoyenneté active. Au fil de l'année, les élèves s'approprient les concepts en les réutilisant à bon escient dans leurs analyses. Leur investissement en EMC souligne leur savoir-faire dans l'utilisation des notions déjà étudiées pour qualifier leur espace public ou pour argumenter en faveur d'un souhait de réaménagement particulier. Enfin, l'observation de la classe lors des débats réalisés en EMC, à l'issue du jeu de rôle, montre qu'en tant qu'habitants, les élèves sont des lecteurs, capables d'ausculter la société à laquelle ils appartiennent. Leurs discours permettent de décrypter leurs pratiques sociales et l'intérêt qu'ils montrent à vivre avec d'autres, notamment à travers la recherche de pratiques spatiales n'excluant aucun acteur qui fréquente la place située devant le lycée.

La question de la transversalité est particulièrement importante. Travailler sur la ville tout au long de l'année, avec des temps d'arrêt en EMC permet de vérifier une évolution positive concernant des savoir-être. A partir de l'exercice sur l'espace public urbain, les 
élèves échangent et débattent sur les incivilités devant le lycée. Cet exercice permet de mettre en pratique des notions et des raisonnements vus en cours d'histoire ou de géographie afin de vérifier les acquis. En outre, les activités réalisées en EMC créent le lien entre les temporalités de la ville. Il paraît évident que les apprentissages des élèves passent hors de ce cadre disciplinaire et que l'EMC permet de relier aisément les activités. Les élèves quittent le statisme du cloisonnement disciplinaire pour une transversalité plus efficace et cohérente.

31 Au-delà d'un socle commun à mettre en place, il est intéressant d'inventer des espaces où l'élève est acteur en-dehors d'un carcan disciplinaire. Dès lors, offrir aux élèves une vision globale de compréhension du monde et de ses enjeux contemporains sur des points d'actualité précis s'avère stimulant pour le futur de l'enseignement secondaire (Morin, 1999). On peut envisager de travailler à la fois de manière transversale et en abordant la complexité avec d'autres objets. Par exemple, le Monde peut être envisagé en ce sens. Une expérience est menée depuis plusieurs années où le Monde est abordé dans sa complexité par les élèves. En effet, des lycéens de Première se préparent dans différentes disciplines (langues, SVT, Géographie, Sciences physiques etc.) à échanger sur des sujets d'actualité en représentant un Etat, une ONG ou autre. Le modèle choisi cette année est celui de la COP 25. D'autres expériences comparables peuvent être menées avec succès, en faisant dialoguer les disciplines et en utilisant l'EMC comme lien essentiel. Il serait profitable de multiplier ces expériences dans les établissements secondaires ces prochaines années.

\section{BIBLIOGRAPHIE}

Allemand, S. \& Dagorn, R.-E. \& Vilaça, O. (2005). La Géographie contemporaine. Paris : Le cavalier bleu.

Audigier, F. (1997). Géographie, Histoire, éducation civique : solidarité réelle ou rendez-vous manqué ?. In R. Knafou (Dir.), L'état de la géographie, autoscopie d'une science (pp. 51-54). Paris : Belin.

Audigier, F. (2012). Préface, In L. Fillion (Dir.), Eduquer à la citoyenneté : construire des compétences sociales et civiques (pp. 11-13). Paris : Scérén/Crap.

Braudel, F. (1969). Ecrits sur l'histoire, Paris : Flammarion.

Bulletin Officiel de l'Education Nationale, novembre 2015.

Bulletin Officiel de l'Education Nationale, janvier 2019.

Cartogram, (2002). Cartographier aujourd'hui, les espaces d'aujourd'hui, Paris : DATAR.

Chabot, G. (1948). Les Villes, Aperçu de géographie humaine. Paris : Armand Colin.

Dumont, G.-F. ( 2016). Comment enseigner la ville et l'urbanité? http://histoire-geo.ac-amiens.fr/ 1005-villes-et-urbanite-gerard-francois-dumont.html 
EDUSCOL, (2010). Le développement durable, fil conducteur du programme de géographie de seconde. Ressources pour le lycée général et technologique. https://

cache.media.eduscol.education.fr/file/lycee/73/9/

LyceeGT_Ressources_HGEC_2_Geo_01_DvptDur_FilConducteur_148739.pdf

EDUSCOL, (2010). Les Européens dans le peuplement de la Terre, programme d'histoire de seconde. Ressources pour le lycée général et technologique. https://

cache.media.eduscol.education.fr/file/lycee/76/3/

LyceeGT_Ressources_HGEC_2_Hist_02_T1EuropeensPopulation_mondiale_148763.pdf

Elias, N. 1991 (1987). La société des individus. Paris : Fayard.

Frémont, A. (1986). Sur l'enseignement de la géographie, L'espace Géographique, (1), 6-17.

Gauvard, C. (2016). En ville, même les manants sont libres ! Sciences et avenir Hors-série Vivre la ville au Moyen-Age.

Hartog, F. (2003). Régimes d'historicité. Présentisme et expériences du temps. Paris : Seuil.

Lefebvre, H.( 2000). La production de l'espace. Paris : Anthropos.

Letissier, F. (2002). Cartographie participative à Saint-Denis. Vers une géographie citoyenne, mémoire de DEA. Reims. Université de Reims.

Letissier, F. (2007). Les temps de l'urbanité. Habiter le patrimoine à Paris. Thèse de doctorat. Reims : Université de Reims.

Lévy, J. (1986). La rente ou la valeur. L’Ecole entre deux géographies, L'espace géographique, (1), $17-23$.

Lévy, J.\& Lussault, M. (2003). Dictionnaire de Géographie et de l'espace des sociétés. Paris : Belin

Lévy, J. (2010). La ville est le développement durable, Métropolitiques, https://

www.metropolitiques.eu/La-ville-est-le-developpement.html

Lussault, M. (2007). L'homme spatial. La construction sociale de l'espace humain. Paris : Seuil.

Médam, A. (1998). Ville pour un sociologue. Paris : l'Harmattan.

Morin, E. (2014). Enseigner à vivre. Manifeste pour changer l'éducation. Arles/Paris : ActesSud/Play Bac.

Morin, E. (1999). Les sept savoirs nécessaires à l'éducation du futur. Paris : Éditions du Seuil.

Morin, E. (1990). Introduction à la pensée complexe. Paris : Seuil.

Mumford, L.(1961). La cité à travers l'histoire. Marseille : Argone.

Noizet, H. \& Cattedu, I. (dir.), (2016). Quoi de neuf au Moyen-Âge ? Paris : La Martinière.

Paquot, T. (2009). L'espace public, Paris : La découverte.

Roumégous, M. (2002). Didactique de la géographie. Enjeux, Résistances, innovations. Rennes : PUR.

Scalab, (2004). La vie quotidienne des lieux, échelles de l'habiter. Paris : PUCA.

Tardif, M. \& Lessard, C. (1999). Le Travail enseignant au quotidien. Expérience, interactions humaines et dilemmes professionnels. Québec, Bruxelles : Presses de l'université de Laval/De Boeck.

Thémine, J.-F. (2006). Enseigner la géographie : un métier qui s'apprend. Paris : Hachette éducation.

Veyret, Y.\& Le Goix, R. (2011). Atlas des villes durables. Paris : Autrement. 
Vezier, A. (2013). L'apprentissage par problématisation, un nouveau modèle pour interroger la textualité des savoirs historiques scolaires. Recherches en Didactiques-Les Cahiers Théodile, 11-28.

\section{NOTES}

1. Programme d'Histoire et de géographie en classe de seconde générale et technologique datant de 2010 et de 2019.

2. Tout au long de mes années de recherche et d'enseignement dans le supérieur, je me suis intéressée à la construction de l'espace urbain, en tant qu'objet social. J'ai notamment travaillé sur une grille d'analyse et de lecture de l'espace public urbain, en tant qu'échelle de prédilection pour appréhender une ville. J'ai également travaillé sur les logiques de conservation et de changement urbains avec différents acteurs et sur différents terrains.

3. Les indications sont données à titre indicatif. Bien que ne l'ayant pas réalisé, Il semble possible de mettre en place une telle démarche avec des élèves de première en l'adaptant aux nouveaux programmes.

4. Thème 2 «L'invention de la citoyenneté dans le monde antique "

5. Dans le cas présent, une photographie personnelle avait été donnée aux élèves. Il s'agissait d'une photographie d'une place urbaine prise à Reims.

6. Consignes données à partir d'une expérience de cartographie participative réalisée avec un groupe d'habitants de Saint-Denis, dans le cadre du programme de recherche CartoGram-Datar.

7. En référence à une analyse des espaces publics urbains réalisée dans le cadre du programme de recherche Scalab pour le PUCA.

8. Les villes du territoire français sont exclues car l'espace français est réservé au programme de première.

9. Les critères proposés par Lévy ont été repris dans leur quasi-totalité (Lévy, 2010). Il s'agit de : la densité, la compacité, la présence d'espaces publics, l'importance des métriques pédestres, la co-présence habitat/emploi, la diversité des activités, la mixité sociologique, la productivité marchande par habitant, le respect de l'environnement naturel.

\section{RÉSUMÉS}

L'enseignement de l'histoire, de la géographie et de l'éducation morale et civique (EMC) dans le secondaire est réalisé par un même enseignant. A la lecture des programmes, il est demandé aux professeurs de respecter " la parité horaire entre l'histoire et la géographie ». Toutefois à partir d'une expérience menée en classe de seconde, nous avons travaillé sur l'objet ville de manière transversale en introduisant la complexité, inhérente à l'espace urbain, tout au long de l'année. Voici une présentation du protocole didactique et de la méthode mise en œuvre.

The teaching of history, geography and Moral and civic education in secondary education is carried out by the same teacher. Reading the programs, teachers have to respect "the hourly parity between history and geography". However from an experiment conducted in first class of 
High School, we worked on the city in a cross-disciplinary approach by introducing complexity. We exectuted a singular framework that we can present.

\section{INDEX}

Mots-clés : ville, lycée, complexité, enseignement transversal, Histoire-Géographie-EMC

Keywords : City, High school, complexity, cross-disciplinary teaching, History-Geography-Moral and Civic education

\section{AUTEUR}

\section{FANNY LETISSIER}

Rhizome de recherche Chôros, Centre International de Recherche Pédagogique (École du sens) 